\title{
HCO mapping of the Horsehead: tracing the illuminated dense molecular cloud surfaces $\star, \star \star$
}

\author{
M. Gerin ${ }^{1}$, J. R. Goicoechea ${ }^{1, \star \star \star}$, J. Pety $^{2,1}$, and P. Hily-Blant ${ }^{3}$ \\ 1 LERMA-LRA, UMR 8112, CNRS, Observatoire de Paris and École Normale Supérieure, 24 Rue Lhomond, 75231 Paris, France \\ e-mail: maryvonne.gerin@lra.ens.fr; jrgoicoechea@fis.ucm.es \\ 2 IRAM, 300 rue de la Piscine, 38406 Grenoble cedex, France \\ e-mail: pety@iram.fr \\ 3 Laboratoire d'Astrophysique, Observatoire de Grenoble, BP 53, 38041 Grenoble Cedex 09, France \\ e-mail: pierre.hilyblant@obs.ujf-grenoble.fr
}

Received 8 September 2008 / Accepted 14 November 2008

\begin{abstract}
Context. Far-UV photons (FUV) strongly affect the physical and chemical state of molecular gas in the vicinity of young massive stars.

Aims. Finding molecular tracers of the presence of FUV radiation fields in the millimeter wavelength domain is desirable because IR diagnostics (for instance PAHs) are not easily accessible along high extinction line-of-sights. Furthermore, gas phase diagnostics provide information on the velocity fields.

Methods. We have obtained maps of the $\mathrm{HCO}$ and $\mathrm{H}^{13} \mathrm{CO}^{+}$ground state lines towards the Horsehead edge at $5^{\prime \prime}$ angular resolution with a combination of Plateau de Bure Interferometer (PdBI) and the IRAM-30 m telescope observations. These maps have been complemented with IRAM-30 m observations of several excited transitions at two different positions.

Results. Bright formyl radical emission delineates the illuminated edge of the nebula, with a faint emission remaining towards the shielded molecular core. Viewed from the illuminated star, the $\mathrm{HCO}$ emission almost coincides with the PAH and CCH emission. HCO reaches a similar abundance to $\mathrm{HCO}^{+}$in the photon dissociation region $(\mathrm{PDR}), \simeq 1-2 \times 10^{-9}$ with respect to $\mathrm{H}_{2}$. To our knowledge, this is the highest $\mathrm{HCO}$ abundance ever measured. Pure gas-phase chemistry models fail to reproduce the observed HCO abundance by $\sim 2$ orders of magnitude, except if reactions of atomic oxygen with carbon radicals abundant in the PDR (i.e., $\mathrm{CH}_{2}$ ) play a significant role in the HCO formation. Alternatively, $\mathrm{HCO}$ could be produced in the PDR by non-thermal processes such as photo-processing of ice mantles and subsequent photo-desorption of either $\mathrm{HCO}$ or $\mathrm{H}_{2} \mathrm{CO}$, and further gas phase photodissociation.

Conclusions. The measured $\mathrm{HCO} / \mathrm{H}^{13} \mathrm{CO}^{+}$abundance ratio is large towards the PDR $(\simeq 50)$, and much lower toward the gas shielded from FUV radiation $(\lesssim 1)$. We propose that high $\mathrm{HCO}$ abundances $\left(\gtrsim 10^{-10}\right)$ together with large $\mathrm{HCO} / \mathrm{H}^{13} \mathrm{CO}^{+}$abundance ratios $(\gtrsim 1)$ are sensitive diagnostics of the presence of active photochemistry induced by FUV radiation.
\end{abstract}

Key words. astrochemistry - ISM: clouds - ISM: molecules - ISM: individual objects: Horsehead nebula - radiative transfer radio lines: ISM

\section{Introduction}

Photodissociation region (PDR) models are used to understand the evolution of far-UV (FUV; $h v<13.6 \mathrm{eV}$ ) illuminated matter both in our Galaxy and in external galaxies. These sophisticated models have been benchmarked Röllig et al. (2007) and are continuously upgraded (e.g., Goicoechea \& Le Bourlot 2007; González-García et al. 2008). Given the large number of physical and chemical processes included in such models, it is necessary to build reference data sets that can be used to test the predictive accuracy of models. Our team has contributed to this goal by providing a series of high resolution interferometric observations of the Horsehead nebula (see Pety et al. 2007b, for

\footnotetext{
* Based on observations obtained with the IRAM Plateau de Bure interferometer and $30 \mathrm{~m}$ telescope. IRAM is supported by INSU/CNRS (France), MPG (Germany), and IGN (Spain).

$\star \star 30 \mathrm{~m}$ maps of the ground state lines are only available in electronic form at the CDS via anonymous ftp to

cdsarc.u-strasbg.fr $(130.79 .128 .5)$ or via

http://cdsweb.u-strasbg.fr/cgi-bin/qcat?J/A+A/494/977

$\star \star \star$ Current address: Centro de Astrobiología. Consejo Superior de Investigaciones Científicas, Madrid, Spain.
}

a summary). Indeed, this source is particularly well suited because of its favorable orientation and geometry, and its moderate distance ( 400 pc; Habart et al. 2005). We have previously studied the carbon (Teyssier et al. 2004; Pety et al. 2005) and sulfur chemistry (Goicoechea et al. 2006) of the nebula, and detected the presence of a cold dense core, with active deuterium fractionation (Pety et al. 2007a).

The formyl radical, HCO, is known to be present in the interstellar medium since the late 1970's Snyder et al. (1976). Snyder et al. (1985) give a detailed description of the HCO structure and discuss the energy diagram for the lowest energy levels. HCO is a bent triatomic asymmetric top with an unpaired electron. $a$-type and $b$-type transitions are allowed, with a stronger dipole moment (1.36 Debye) for the $a$-type transitions Landsberg et al. (1977), which are therefore more easily detectable. The strongest HCO ground state transitions lie at 86.670, 86.708, 86.777 and $86.805 \mathrm{GHz}$, very close to the ground state transition of $\mathrm{H}^{13} \mathrm{CO}^{+}$ and to the first rotationally excited $\mathrm{SiO}$ line. Therefore $\mathrm{HCO}$ can be observed simultaneously with $\mathrm{SiO}$ and $\mathrm{H}^{13} \mathrm{CO}^{+}$. $\mathrm{HCO}$ ground state lines have been detected in the Orion Bar as well as in the dense PDRs NGC 2023, NGC 7023 and S 140 Schilke et al. (2001). From limited mapping, they have shown that HCO is 
Table 1. Observation parameters for the maps shown in Figs. 1 and 5. The projection center of all the maps is $\alpha_{2000}=05^{\mathrm{h}} 40^{\mathrm{m}} 54.27^{\mathrm{s}}, \delta_{2000}=$ $-02^{\circ} 28^{\prime} 00^{\prime \prime}$

\begin{tabular}{|c|c|c|c|c|c|c|c|c|c|c|c|}
\hline Molecule & Transition & $\begin{array}{r}\text { Frequency } \\
\mathrm{GHz}\end{array}$ & Instrument & Config. & $\begin{array}{l}\text { Beam } \\
\text { arcsec }\end{array}$ & $\begin{array}{c}\text { PA } \\
\circ\end{array}$ & $\begin{array}{c}\text { Vel. Resol. } \\
\mathrm{km} \mathrm{s}^{-1}\end{array}$ & $\begin{array}{l}\text { Int. Time } \\
\text { h }\end{array}$ & $\begin{array}{c}T_{\text {sys }} \\
\mathrm{K}\end{array}$ & $\begin{array}{c}\text { Noise }^{b} \\
\text { K }\end{array}$ & Obs. date \\
\hline $\mathrm{H}^{13} \mathrm{CO}^{+}$ & $1-0$ & 86.754288 & PdBI & $C \& D$ & $6.76 \times 4.65$ & 13 & 0.2 & 6.5 & 150 & 0.10 & 2006-2007 \\
\hline $\mathrm{HCO}$ & $1_{0,1} 3 / 2,2-0_{0,0} 1 / 2,1$ & 86.670760 & PdBI & $C \& D$ & $6.69 \times 4.39$ & 16 & 0.2 & 6.5 & 150 & 0.09 & 2006-2007 \\
\hline $\mathrm{CCH}$ & $1,3 / 2(2)-0,1 / 2(1)$ & 87.316925 & PdBI & $C \& D$ & $7.24 \times 4.99$ & 54 & 0.2 & 6.9 & 130 & 0.07 & 2002-2003 \\
\hline
\end{tabular}

${ }^{a}$ On-source time computed as if the source were always observed with 6 antennae. ${ }^{b}$ The noise values quoted here are the values at the mosaic phase center (Mosaic noise is inhomogeneous due to primary beam correction; it steeply increases at the mosaic edges).

\begin{tabular}{|c|c|c|c|c|c|c|c|c|c|c|c|c|}
\hline Molecule & Transition & $\begin{array}{r}\text { Frequency } \\
\mathrm{GHz} \\
\end{array}$ & Instrument & \# Pix. & $F_{\text {eff }}$ & $B_{\text {eff }}$ & $\begin{array}{l}\text { Resol. } \\
\text { arcsec }\end{array}$ & $\begin{array}{l}\text { Resol. } \\
\mathrm{km} \mathrm{s}^{-1}\end{array}$ & $\begin{array}{l}\text { Int. Time }{ }^{a} \\
\mathrm{~h}\end{array}$ & $\begin{array}{c}T_{\text {sys }} \\
\mathrm{K}\end{array}$ & $\begin{array}{c}\text { Noise } \\
\mathrm{mK}\end{array}$ & Obs. date \\
\hline $\mathrm{H}^{13} \mathrm{CO}^{+}$ & & 86.754288 & & 2 & & 0.7 & 28.4 & 0.2 & & 133 & 69 & \\
\hline $\mathrm{HCO}$ & 32 & 760 & & 2 & 0.95 & 0.78 & 29. & 0. & & 133 & 63 & 007 \\
\hline $\mathrm{HCO}$ & & 8360 & & 2 & 0.95 & 0.78 & 29.9 & 0. & & 133 & 63 & 2007 \\
\hline $\mathrm{HCO}$ & $1 / 2,1$ & 86.777460 & B100 & 2 & 0.95 & 0.78 & 29.9 & 0. & $2.6 / 5.0$ & 133 & 66 & 2006-2007 \\
\hline $\mathrm{HCO}$ & $1_{0,1} 1 / 2,0-0_{0,0} 1 / 2,1$ & 86.805780 & $30 \mathrm{~m} / \mathrm{AB} 100$ & 2 & 0.95 & 0.78 & 29.9 & 0.2 & $2.6 / 5.0$ & 133 & 66 & 2006-2007 \\
\hline
\end{tabular}

${ }^{a}$ Two values are given for the integration time: the on-source time and the telescope time.

Table 2. Observation parameters for the HCO deep integrations shown in Fig. 1. Associated transitions can be found in Table 3 . The RA and Dec offsets are computed with reference to $\alpha_{2000}=05^{\mathrm{h}} 40^{\mathrm{m}} 54.27^{\mathrm{s}}, \delta_{2000}=-02^{\circ} 28^{\prime} 00^{\prime \prime}$. The positions are also given in the coordinate system used to display the maps in Figs. 1 and 5. In this coordinate system, maps are rotated by $14^{\circ}$ counter-clockwise around the projection center, located at $(\delta x, \delta y)=\left(20^{\prime \prime}, 0^{\prime \prime}\right)$, to bring the illuminated star direction in the horizontal direction and the horizontal zero has been set at the PDR edge.

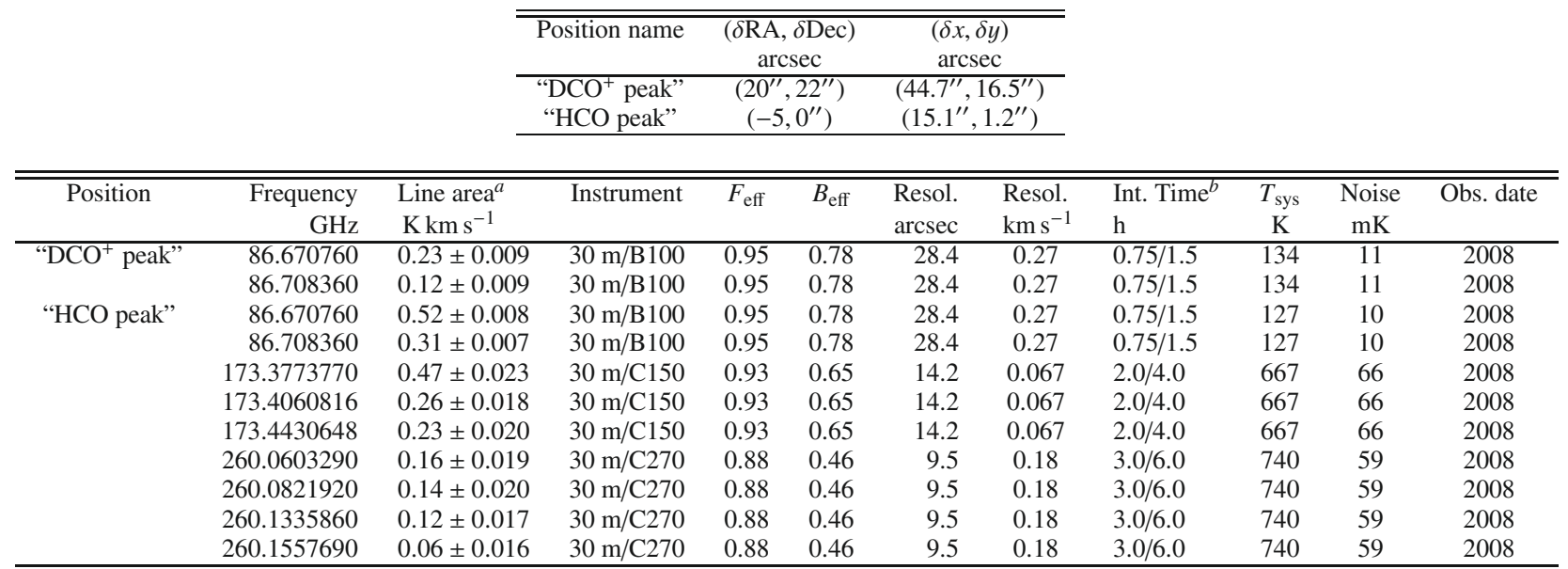

${ }^{a}$ Values obtained from Gaussian fits performed on the spectra using the main beam temperature scale. ${ }^{b}$ Two values are given for the integration time: the on-source time and the telescope time.

sharply peaked in the Orion Bar PDR, confirming earlier suggestions that $\mathrm{HCO}$ is a tracer of the cloud illuminated interfaces de Jong et al. (1980). García-Burillo et al. (2002) have mapped $\mathrm{HCO}$ and $\mathrm{H}^{13} \mathrm{CO}^{+}$in the nearby galaxy $\mathrm{M} 82$. HCO, CO and the ionized gas present a nested ring morphology, with the HCO peaks being located further out compared to $\mathrm{CO}$ and the ring of H II regions. The chemistry of HCO is not well understood. Schilke et al. (2001) concluded that it is extremely difficult to understand the observed HCO abundance in PDRs with gas phase chemistry alone. As a possible way out, they tested the production of $\mathrm{HCO}$ by the photodissociation of formaldehyde. In this model, $\mathrm{H}_{2} \mathrm{CO}$ is produced in grain mantles, and released by nonthermal photo-desorption in the gas phase in the PDR. However, even with this favorable hypothesis, the model cannot reproduce the abundance and spatial distribution of $\mathrm{HCO}$ because the photo-production is most efficient at an optical depth of a few magnitudes where the photodissociation becomes less effective.

In this paper, we present maps of the formyl radical ground state lines at high angular resolution towards the Horsehead nebula, and the detection of higher energy level transitions towards two particular lines of sights, one in the PDR region and the other in the associated dense core. These observations enable us to accurately study the HCO spatial distribution and abundance. We present the observations and data reduction in Sect. 2, while the results and HCO abundance are given in Sect. 3, and the discussion of HCO chemistry and PDR modeling is given in Sect. 4.

\section{Observations and data reduction}

Tables 1 and 2 summarize the observation parameters for the data obtained with the IRAM PdBI and $30 \mathrm{~m}$ telescopes. The HCO ground state lines were observed simultaneously with $\mathrm{H}^{13} \mathrm{CO}^{+}$and $\mathrm{SiO}$. Frequency-switched, on-the-fly maps of the $\mathrm{H}^{13} \mathrm{CO}^{+} J=1-0$ and $\mathrm{HCO}$ ground state lines (see Fig. 5), obtained at the IRAM-30m using the A100 and B100 $3 \mathrm{~mm}$ receivers ( $\sim 7 \mathrm{~mm}$ of water vapor) were used to produce the short-spacings needed to complement a 7-field mosaic acquired with the $6 \mathrm{PdBI}$ antennae in the $\mathrm{CD}$ configuration (baseline lengths from 24 to $176 \mathrm{~m}$ ). The whole PdBI data set will be comprehensively described in a forthcoming paper studying the 

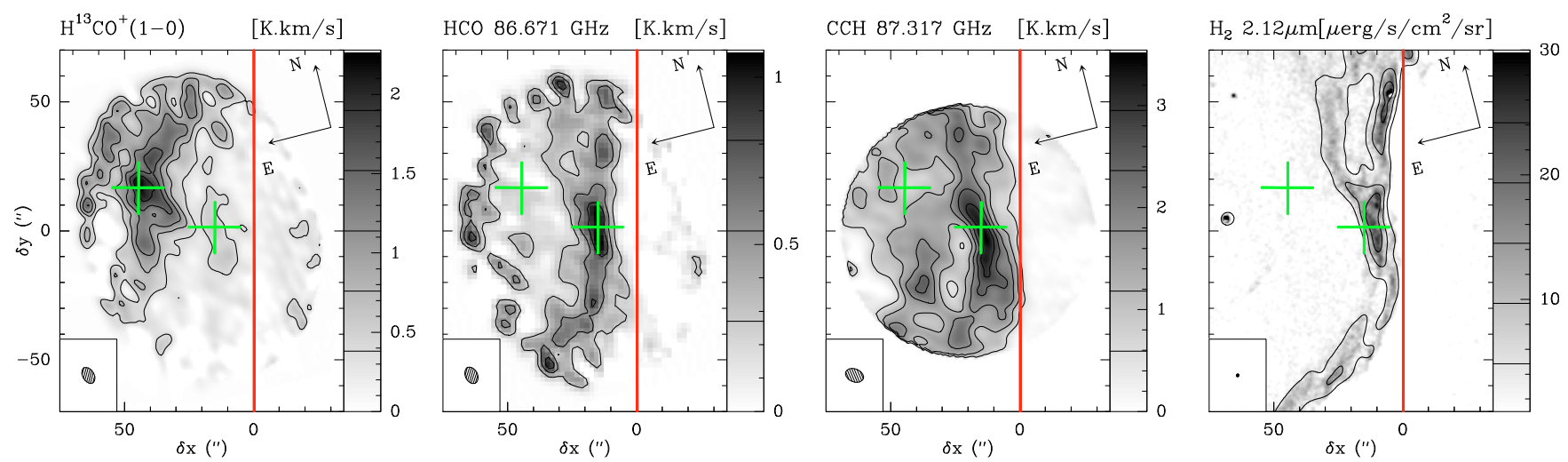

Fig. 1. High angular resolution maps of the integrated intensity of $\mathrm{H}^{13} \mathrm{CO}^{+}, \mathrm{HCO}, \mathrm{CCH}$ and vibrationally excited $\mathrm{H}_{2}$ emission. $\mathrm{H}^{13} \mathrm{CO}^{+}$and $\mathrm{HCO}$ have been observed simultaneously, both with the IRAM-30 m and IRAM-PdBI. Maps have been rotated by $14^{\circ}$ counter-clockwise around the projection center, located at $(\delta x, \delta y)=\left(20^{\prime \prime}, 0^{\prime \prime}\right)$, to bring the illuminated star direction in the horizontal direction and the horizontal zero has been set at the PDR edge. The emission of all lines is integrated between 10.1 and $11.1 \mathrm{~km} \mathrm{~s}^{-1}$. Displayed integrated intensities are expressed in the main beam temperature scale. Contour levels are displayed on the grey scale lookup tables. The red vertical line shows the PDR edge and the green crosses show the positions ( $\mathrm{DCO}^{+}$and $\mathrm{HCO}$ peaks) where deep integrations have been performed at IRAM-30 m (see Fig. 2). The $\mathrm{H}_{2}$ map is taken from Habert et al. (2005).

fractional ionization across the Horsehead edge (Goicoechea et al. 2009). The $\mathrm{CCH}$ data shown in Fig. 1 have been extensively described in Pety et al. (2005). The high resolution HCO $1_{0,1}-0_{0,0}$ data are complemented by observations of the $2_{0,2}-1_{0,1}$ and $3_{0,3}-2_{0,2}$ multiplets with the IRAM $30 \mathrm{~m}$ telescope centered on the PDR and the dense core. To obtain those deep integration spectra, we used the position switching observing mode. The on-off cycle duration was $1 \mathrm{~min}$ and the off-position offsets were $(\delta \mathrm{RA}, \delta \mathrm{Dec})=\left(-100^{\prime \prime}, 0^{\prime \prime}\right)$, i.e. the H II region ionized by $\sigma$ Ori and free of molecular emission. Position accuracy is estimated to be about $3^{\prime \prime}$ for the $30 \mathrm{~m}$ data and less than $0.5^{\prime \prime}$ for the PdBI data.

The data processing was done with the GILDAS $^{1}$ softwares (Pety 2005b). The IRAM-30 m data were first calibrated to the $T_{\mathrm{A}}^{*}$ scale using the chopper wheel method (Penzias \& Burrus 1973), and finally converted to main beam temperatures $\left(T_{\mathrm{mb}}\right)$ using the forward and main beam efficiencies $\left(F_{\text {eff }}\right.$ $\left.\& B_{\text {eff }}\right)$ displayed in Table 2 . The resulting amplitude accuracy is $\sim 10 \%$. Frequency-switched spectra were folded using the standard shift-and-add method, after baseline subtraction. The resulting spectra were finally gridded through convolution by a Gaussian. Position-switched spectra were co-added before baseline subtraction. Interferometric data and short-spacing data were merged before imaging and deconvolution of the mosaic, using standard techniques of GILDAS (see e.g. Pety et al. 2005, for details).

\section{Results and discussion}

\subsection{Spatial distribution}

Figure 1 shows a map of the integrated intensity of the strongest $\mathrm{HCO}$ line at $86.671 \mathrm{GHz}$, of the $\mathrm{H}^{13} \mathrm{CO}^{+} J=1-0$ line and of the strongest $\mathrm{CCH}$ line at $87.317 \mathrm{GHz}$. Figure 2 displays high signal-to-noise ratio spectra of several hyperfine components of three $\mathrm{HCO}$ rotational transitions towards the $\mathrm{HCO}$ and the $\mathrm{DCO}^{+}$emission peaks.

Most of the formyl radical emission is concentrated in a narrow structure, delineating the edge of the Horsehead nebula.

\footnotetext{
${ }^{1}$ See http://wWw. iram.fr/IRAMFR/GILDAS for more information about the GILDAS softwares.
}

Low level emission is however detected throughout the nebula, including towards the dense core identified by its strong $\mathrm{DCO}^{+}$ and $\mathrm{H}^{13} \mathrm{CO}^{+}$emission Pety et al. (2007a). The HCO emission is resolved by our PdBI observations. From 2-dimensional Gaussian fits of the image, we estimate that the emission width is $\sim 13 \pm 4^{\prime \prime}$ in the plane of the sky. The $\mathrm{H}^{13} \mathrm{CO}^{+}$emission shows a different pattern: most of the signal is associated with the dense core behind the photodissociation front, and faint $\mathrm{H}^{13} \mathrm{CO}^{+}$emission detected in the illuminated edge. The $\mathrm{CCH}$ emission pattern is less extreme than $\mathrm{HCO}$, but shows a similar enhancement in the PDR.

In summary, the morphology of the HCO emission is reminiscent of the emission of the PDR tracers, either the PAH emission (Abergel et al. 2002) or the emission of small hydrocarbons, which is strongly enhanced towards the PDR (Teyssier et al. 2004; Pety et al. 2005). In contrast, the HCO emission becomes very faint where the gas is dense and shielded from FUV radiation. These regions are associated with bright $\mathrm{DCO}^{+}$ and $\mathrm{H}^{13} \mathrm{CO}^{+}$emission (Pety et al. 2007a). Our maps therefore confirm that $\mathrm{HCO}$ is a PDR species.

\subsection{Column densities and abundances}

\subsubsection{Radiative transfer models of $\mathrm{HCO}$ and $\mathrm{H}^{13} \mathrm{CO}^{+}$}

Einstein coefficients and upper level energies of the studied $\mathrm{HCO}$ and $\mathrm{H}^{13} \mathrm{CO}^{+}$lines are given in Table 3. As no collisional crosssections with $\mathrm{H}_{2}$ nor He have been calculated for $\mathrm{HCO}$ so far, we have computed the HCO column densities assuming a single excitation temperature $T_{\mathrm{ex}}$ for all transitions. Nevertheless our calculation takes into account thermal, turbulent and opacity broadening as well as the cosmic microwave background and line opacity Goicoechea et al. (2006). For $\mathrm{H}^{13} \mathrm{CO}^{+}$, detailed non-local and non-LTE excitation and radiative transfer calculations have been performed using the same approach as in our previous PdBI CS and $\mathrm{C}^{18} \mathrm{O}$ line analysis (see Appendix in Goicoechea et al. 2006). $\mathrm{H}^{13} \mathrm{CO}^{+}-\mathrm{H}_{2}$ collisional rate coefficients were adapted from those of Flower (1999) for $\mathrm{HCO}^{+}$, and specific $\mathrm{H}^{13} \mathrm{CO}^{+}$-electron rates where kindly provided by Faure \& Tennyson (in prep.). 


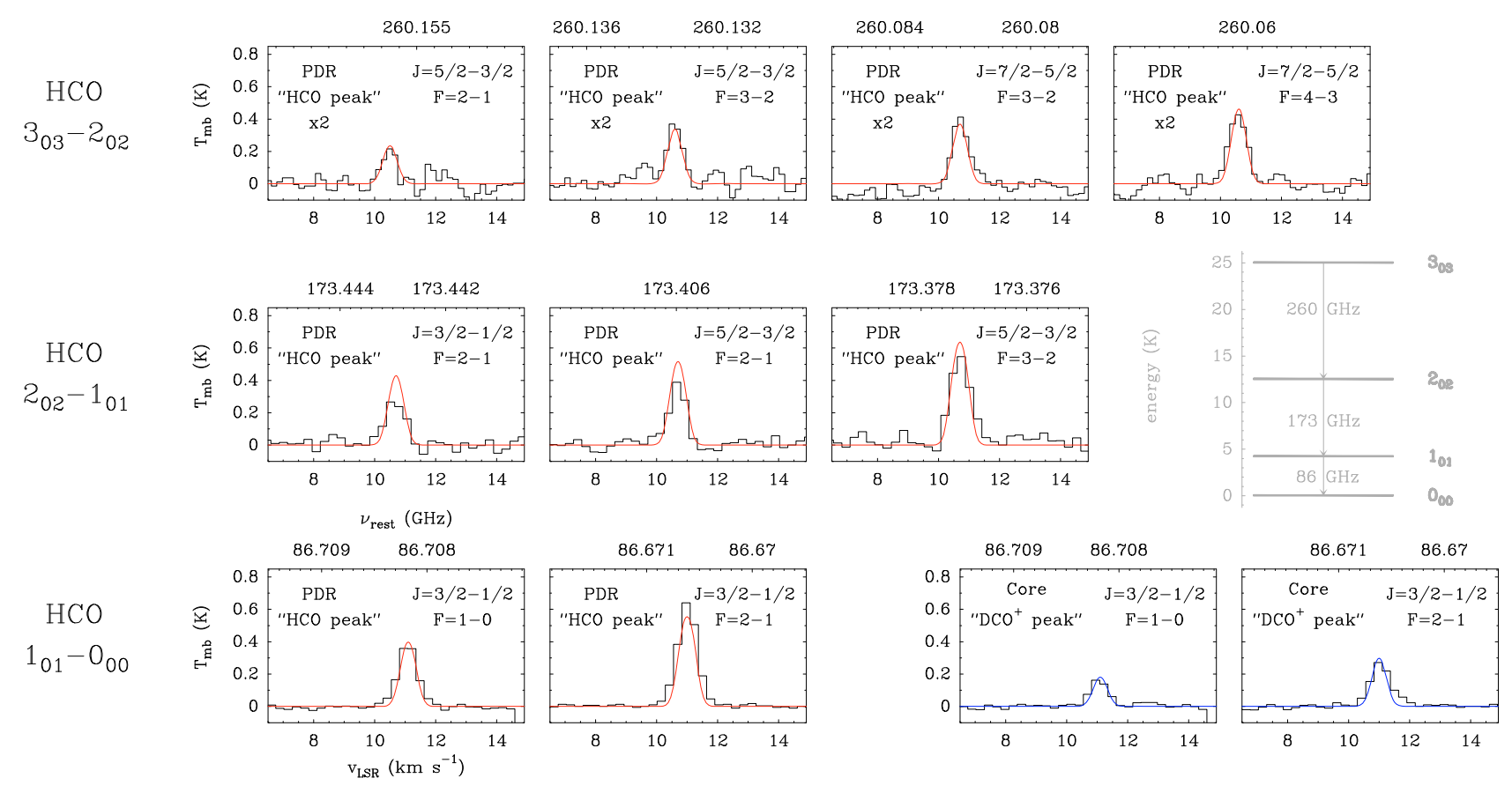

Fig. 2. IRAM-30 m observations (histograms) of several HCO hyperfine components of the $1_{01}-0_{00}, 2_{02}-1_{01}$ and $3_{03}-2_{02}$ rotational transitions towards the PDR ("HCO peak") and towards the dense core ("DCO ${ }^{+}$peak") Pety et al. (2007a). Solid lines are single- $T_{\text {ex }}$ radiative transfer models of the PDR-filament (red curves) and line-of-sight cloud surface (blue curves). A sketch of the HCO rotational energy levels is also shown (right corner).

Table 3. Einstein coefficients and upper level energies.

\begin{tabular}{ccrcr}
\hline \hline Molecule & $\begin{array}{r}\text { Transition } \\
J, F-J^{\prime}, F^{\prime}\end{array}$ & $\begin{array}{r}\text { Frequency } \\
\mathrm{GHz}\end{array}$ & $\begin{array}{c}A_{i j} \\
\left(\mathrm{~s}^{-1}\right)\end{array}$ & $\begin{array}{r}E_{\text {up }} \\
(\mathrm{K})\end{array}$ \\
\hline $\mathrm{HCO}$ & $1_{01}-0_{00}$ & & & \\
$3 / 2,2-1 / 2,1$ & 86.670760 & $4.69 \times 10^{-6}$ & 4.2 \\
& $3 / 2,1-1 / 2,0$ & 86.708360 & $4.60 \times 10^{-6}$ & 4.2 \\
$1 / 2,1,1 / 2,0$ & 86.777460 & $4.61 \times 10^{-6}$ & 4.2 \\
$1 / 2,0-1 / 2,1$ & 86.805780 & $4.71 \times 10^{-6}$ & 4.2 \\
$2_{02}-1_{01}$ & & & \\
$5 / 2,3-3 / 2,2$ & 173.3773770 & $4.51 \times 10^{-5}$ & 12.5 \\
& $5 / 2,2-3 / 2,1$ & 173.4060816 & $4.43 \times 10^{-5}$ & 12.5 \\
$3 / 2,2-1 / 2,1$ & 173.4430648 & $3.39 \times 10^{-5}$ & 12.5 \\
$3_{03}-202$ & & & \\
$7 / 2,4-5 / 2,3$ & 260.0603290 & $1.63 \times 10^{-4}$ & 25.0 \\
& $7 / 2,3-5 / 2,2$ & 260.0821920 & $1.61 \times 10^{-4}$ & 25.0 \\
$5 / 2,3-3 / 2,2$ & 260.1335860 & $1.45 \times 10^{-4}$ & 25.0 \\
& $5 / 2,2-3 / 2,1$ & 260.1557690 & $1.37 \times 10^{-4}$ & 25.0 \\
\hline $\mathrm{H}^{13} \mathrm{CO}^{+}$ & $J=1-0$ & 86.754288 & $3.2 \times 10^{-5}$ & 4.2 \\
& $J=3-2$ & 260.2553390 & $1.3 \times 10^{-3}$ & 25.0 \\
\hline
\end{tabular}

The line frequencies and intensities were extracted from the JPL Pickett et al. (1998) and CDMS (Müller et al. 2001, 2005) molecular spectroscopy data bases for $\mathrm{HCO}$ and $\mathrm{H}^{13} \mathrm{CO}^{+}$respectively.

\subsubsection{Structure of the PDR in $\mathrm{HCO}$ and $\mathrm{H}^{13} \mathrm{CO}^{+}$}

For more insight into the spatial variation of the $\mathrm{HCO}$ and $\mathrm{H}^{13} \mathrm{CO}^{+}$column densities and abundances, we have analyzed a cut through the PDR, centered on the "HCO peak" at $\delta y=0$ " (see Fig. 3). The cut clearly shows that HCO is brighter than $\mathrm{H}^{13} \mathrm{CO}^{+}$in the PDR and vice-versa in the dense core. Taking into account the different level degeneracies of both transitions (a factor of 2.4) and the fact that the associated Einstein

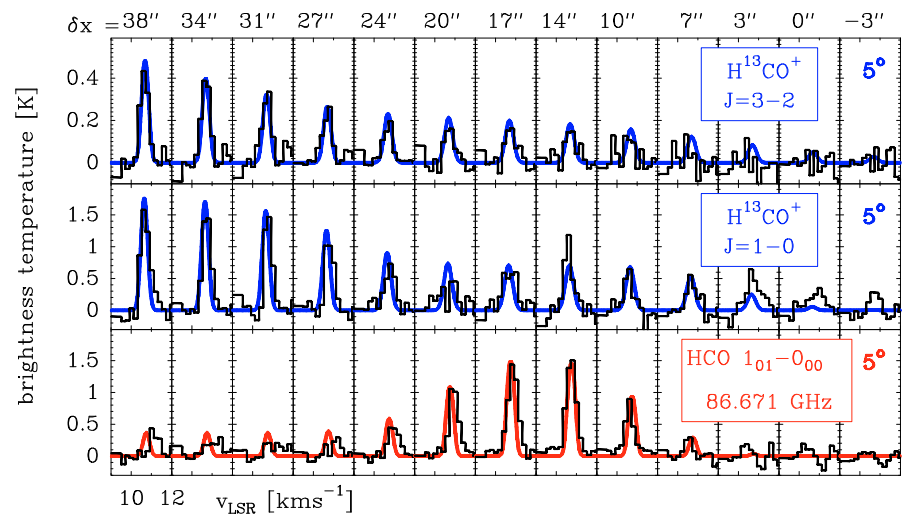

Fig. 3. Observations along a horizontal cut through "the HCO peak" (histograms). The $\mathrm{H}^{13} \mathrm{CO}^{+} J=1-0$ and $\mathrm{HCO} 1_{01}-0_{00}$ lines were mapped with the PdBI at an angular resolution of $6.8^{\prime \prime}$, whereas the $\mathrm{H}^{13} \mathrm{CO}^{+} J=3-2$ line was mapped with HERA-30 m (and smoothed to a spatial resolution of $13.5^{\prime \prime}$ ). Radiative transfer models of an edge-on cloud with a line of sight extinction of $A_{\mathrm{V}}=20$, inclined $5^{\circ}$ relative to the line of sight for $\mathrm{HCO}$ (red curve), and $\mathrm{H}^{13} \mathrm{CO}^{+}$(blue curves) are shown. The single- $T_{\text {ex }}$ HCO model assumes a $12^{\prime \prime}$ width filament with a column density of $3.2 \times 10^{13} \mathrm{~cm}^{-2}$, while $N(\mathrm{HCO})$ is $4.6 \times 10^{12} \mathrm{~cm}^{-2}$ behind the filament. The $\mathrm{H}^{13} \mathrm{CO}^{+}$model assumes a constant density of $n\left(\mathrm{H}_{2}\right)=5 \times 10^{4} \mathrm{~cm}^{-3}$ with $T_{\mathrm{k}}=60 \mathrm{~K}$ and $N\left(\mathrm{H}^{13} \mathrm{CO}^{+}\right)=5.8 \times 10^{11} \mathrm{~cm}^{-2}$ for $\delta x<35^{\prime \prime}$; and $T_{\mathrm{k}}=10 \mathrm{~K}$ and $N\left(\mathrm{H}^{13} \mathrm{CO}^{+}\right)=7.6 \times 10^{11} \mathrm{~cm}^{-2}$ for $\delta x>35^{\prime \prime}$. Modeled line profiles have been convolved with an appropriate Gaussian beam corresponding to each PdBI synthesized beam or $30 \mathrm{~m}$ main beam resolution.

coefficients $A_{i j}$ differ by a factor $\sim 8$ (due to the different permanent dipole moments, see Table 3$), N\left(\mathrm{H}^{13} \mathrm{CO}^{+}\right)$must be significantly lower than $N(\mathrm{HCO})$ towards the PDR.

We modeled the PDR as an edge-on cloud inclined by $\sim 5^{\circ}$ relative to the line-of-sight. We have chosen a cloud depth 
of $\sim 0.1 \mathrm{pc}$, which implies an extinction of $A_{\mathrm{V}} \simeq 20$ mag for the considered densities towards the "HCO peak". These parameters are the best geometrical description of the Horsehead PDR-edge (e.g., Habart et al. 2005) and also reproduce the observed $1.2 \mathrm{~mm}$ continuum emission intensity. The details of this modeling will be presented in Goicoechea et al. (2009). In the following, we describe in detail the determination of the column densities and abundances for two particular positions, namely the "HCO peak" and the "DCO ${ }^{+}$peak" (offsets relative to the map center can be found in Table 2).

\subsubsection{HCO column densities}

We used the three detected rotational transitions of HCO (each with several hyperfine components, see Fig. 2) to estimate the $\mathrm{HCO}$ column densities in the direction of the "HCO" peak. We have taken into account the varying beam dilution factors of the $\mathrm{HCO}$ emission at the "HCO peak" by modeling the HCO emission as a Gaussian filament of $\sim 12^{\prime \prime}$ width in the $\delta x$ direction, and infinite in the $\delta y$ direction. The filling factors at 260, 173 and $87 \mathrm{GHz}$ are thus $\sim 0.8,0.6$ and 0.4 , respectively.

A satisfactory fit of the IRAM-30 m data towards the "HCO peak" is obtained for $T_{\text {ex }} \simeq 5 \mathrm{~K}$ and a turbulent velocity dispersion of $\sigma=0.225 \mathrm{~km} \mathrm{~s}^{-1}(F W H M=2.355 \times \sigma)$. Line profiles are reproduced for $N(\mathrm{HCO})=3.2 \times 10^{13} \mathrm{~cm}^{-2}$ (see red solid curves in Fig. 2). The most intense HCO lines at 86.67 and $173.38 \mathrm{GHz}$ become marginally optically thick at this column density $(\tau \gtrsim 1)$. Therefore, opacity corrections need to be taken into account. We checked that the low value of $T_{\mathrm{ex}}$ (subthermal excitation as $T_{\mathrm{k}} \simeq 60 \mathrm{~K}$ ) is consistent with detailed excitation calculations carried out for $\mathrm{H}^{13} \mathrm{CO}^{+}$in the PDR which are described below.

Because the HCO signals are weaker towards the " $\mathrm{DCO}^{+}$peak", we only detected 2 hyperfine components of the $1_{01}-0_{00}$ transition. Assuming extended emission and the same excitation temperature as for the "HCO peak", $5 \mathrm{~K}$, we fit the observed lines with a column density of $4.6 \times 10^{12} \mathrm{~cm}^{-2}$ (blue solid lines in Fig. 2). Both HCO lines are optically thin at this position. This simple analysis shows that the HCO column density is $\sim 7$ times larger at the "HCO peak" in the PDR, than towards the dense cold core.

\subsection{4. $\mathrm{H}^{13} \mathrm{CO}^{+}$column densities}

Both the $\mathrm{H}^{13} \mathrm{CO}^{+} J=3-2$ and 1-0 line profiles at the "HCO peak" are fitted with $n\left(\mathrm{H}_{2}\right) \simeq 5 \times 10^{4} \mathrm{~cm}^{-3}, T_{\mathrm{k}} \simeq 60 \mathrm{~K}$ and $\mathrm{e}^{-} / \mathrm{H} \simeq 5 \times 10^{-5}$ (as predicted by the PDR models below). The required column density is $N\left(\mathrm{H}^{13} \mathrm{CO}^{+}\right)=5.8 \times 10^{11} \mathrm{~cm}^{-2}$. For those conditions, the excitation temperature, $T_{\mathrm{ex}}$, of the $J=3-2$ transition varies from $\simeq 4$ to $6 \mathrm{~K}$, which supports the single- $T_{\text {ex }}$ models of HCO. Both $\mathrm{H}^{13} \mathrm{CO}^{+}$lines are optically thin towards the "HCO peak".

The $\mathrm{H}^{13} \mathrm{CO}^{+}$line emission towards the " $\mathrm{DCO}^{+}$peak" has been studied by Pety et al. (2007a). Both $\mathrm{H}^{13} \mathrm{CO}^{+}$lines are moderately optically thick towards the core, and the $\mathrm{H}^{13} \mathrm{CO}^{+}$column density is $N\left(\mathrm{H}^{13} \mathrm{CO}^{+}\right) \simeq 5.0 \times 10^{12} \mathrm{~cm}^{-2}$, which represents an enhancement of nearly one order of magnitude relative to the PDR. According to our $1.2 \mathrm{~mm}$ continuum map, the extinction towards the core is $A_{\mathrm{V}} \gtrsim 30 \mathrm{mag}$ compared to $20 \mathrm{mag}$ in the PDR. The $\mathrm{H}^{13} \mathrm{CO}^{+}$column density enhancement therefore corresponds to a true abundance enhancement.
Table 4. Inferred column densities and abundances with respect to molecular hydrogen, e.g. $\chi(\mathrm{X})=N(\mathrm{X}) / N\left(\mathrm{H}_{2}\right)$.

\begin{tabular}{lccc}
\hline \hline Molecule & Method & HCO peak & DCO $^{+}$peak \\
\hline$N\left(\mathrm{H}_{2}\right)\left[\mathrm{cm}^{-2}\right]$ & $1.2 \mathrm{~mm}$ cont. & $1.9 \times 10^{22}$ & $2.9 \times 10^{22}$ \\
\hline$N(\mathrm{HCO})\left[\mathrm{cm}^{-2}\right]$ & $T_{\mathrm{ex}}=5 \mathrm{~K}$ & $3.2 \times 10^{13}$ & $4.6 \times 10^{12}$ \\
$N\left(\mathrm{H}^{13} \mathrm{CO}^{+}\right)\left[\mathrm{cm}^{-2}\right]$ & Full excitation & $5.8 \times 10^{11}$ & $5.0 \times 10^{12 *}$ \\
$N\left(\mathrm{HCO}^{+}\right)\left[\mathrm{cm}^{-2}\right]$ & ${ }^{12} \mathrm{C} /{ }^{13} \mathrm{C}=60$ & $3.5 \times 10^{13}$ & $3.0 \times 10^{14}$ \\
\hline$\chi\left(\mathrm{HCO}^{+}\right)$ & & $1.7 \times 10^{-9}$ & $1.6 \times 10^{-10 \dagger}$ \\
$\chi\left(\mathrm{H}^{13} \mathrm{CO}^{+}\right)$ & & $3.1 \times 10^{-11}$ & $1.7 \times 10^{-10}$ \\
$\chi\left(\mathrm{HCO}^{+}\right)$ & & $1.8 \times 10^{-9}$ & $1.0 \times 10^{-8}$ \\
\hline
\end{tabular}

${ }^{*}$ Pety et al. (2007a).

$\dagger 1.7 \times 10^{-9}$ if $\mathrm{HCO}$ arises only from the cloud surface $\left(A_{\mathrm{V}} \simeq 3\right)$.

\subsubsection{Comparison of $\mathrm{HCO}$ and $\mathrm{H}^{13} \mathrm{CO}^{+}$abundances}

Table 4 summarizes the inferred $\mathrm{HCO}$ and $\mathrm{H}^{13} \mathrm{CO}^{+}$column densities and abundances towards the 2 selected positions: the "HCO peak" in the PDR and the "DCO ${ }^{+}$peak" in the FUVshielded core. Both species exhibit strong variations of their column densities and abundances relative to $\mathrm{H}_{2}$ between the PDR and the shielded region. In the PDR, we found that both the $\mathrm{HCO}$ abundance relative to $\mathrm{H}_{2}\left(\chi(\mathrm{HCO}) \simeq 1-2 \times 10^{-9}\right)$ and the $\mathrm{HCO} / \mathrm{H}^{13} \mathrm{CO}^{+}$column density ratio $(\approx 50)$ are high. These figures are higher than all previously published measurements (at lower angular resolution). Besides, the formyl radical and $\mathrm{HCO}^{+}$ reach similar abundances in the PDR.

The situation is reversed towards the " $\mathrm{DCO}^{+}$peak", i.e. the observed $\mathrm{HCO} / \mathrm{H}^{13} \mathrm{CO}^{+}$column density ratio is lower $(\approx 1)$ than towards the "HCO peak". Nevertheless, while the bulk of the observed $\mathrm{H}^{13} \mathrm{CO}^{+}$emission arises from cold and shielded gas, the origin of $\mathrm{HCO}$ emission is less clear. HCO could either (i) coexist with $\mathrm{H}^{13} \mathrm{CO}^{+}$or (ii) arise predominantly from the line-ofsight cloud surface. In the former case, our observations show that the HCO abundance drops by one order of magnitude between the PDR and the dense core environment. However, it is possible that the abundance variation is even more pronounced, if the detected $\mathrm{HCO}$ emission arises from the line of sight cloud surface. We have estimated the depth of the cloud layer, assuming that HCO keeps the "PDR abundance" in this foreground layer: a cloud surface layer of $A_{\mathrm{V}} \simeq 3$ (illuminated by the mean FUV radiation field around the region) also reproduces the observed HCO lines towards the cold and dense core (blue solid lines in Fig. 2).

In this case, both the $\mathrm{HCO}$ abundance and the $\mathrm{HCO} / \mathrm{H}^{13} \mathrm{CO}^{+}$ abundance ratio in the dense core itself will be even lower than listed in Table 4 . We have tried to discriminate between the scenarios by comparing the HCO $1_{01}-0_{00}(J=3 / 2-1 / 2$, $F=2-1)$ and $\mathrm{H}^{13} \mathrm{CO}^{+} J=1-0$ line profiles towards this position. Both lines have been observed simultaneously with the IRAM-30 m telescope. Because of their very similar frequencies $(\sim 86.7 \mathrm{GHz})$, the beam profile and angular resolution is effectively the same. In this situation, any difference in the measured linewidths reflects real differences in the gas kinematics and turbulence of the regions where the line profiles are formed. Gaussian fits of the $\mathrm{HCO}$ and $\mathrm{H}^{13} \mathrm{CO}^{+}$lines towards "the $\mathrm{DCO}^{+}$ peak" provides line widths of $\Delta v(\mathrm{HCO})=0.81 \pm 0.06 \mathrm{~km} \mathrm{~s}^{-1}$ and $\Delta v\left(\mathrm{H}^{13} \mathrm{CO}^{+}\right)=0.60 \pm 0.01 \mathrm{~km} \mathrm{~s}^{-1}$. Therefore, even if the $\mathrm{H}^{13} \mathrm{CO}^{+} J=1-0$ lines are slightly broadened by opacity and do not represent the true line of sight velocity dispersion, HCO lines are broader at the $3 \sigma$ level of confidence. This remarkable difference supports the scenario (ii) where the $\mathrm{H}^{13} \mathrm{CO}^{+}$line 

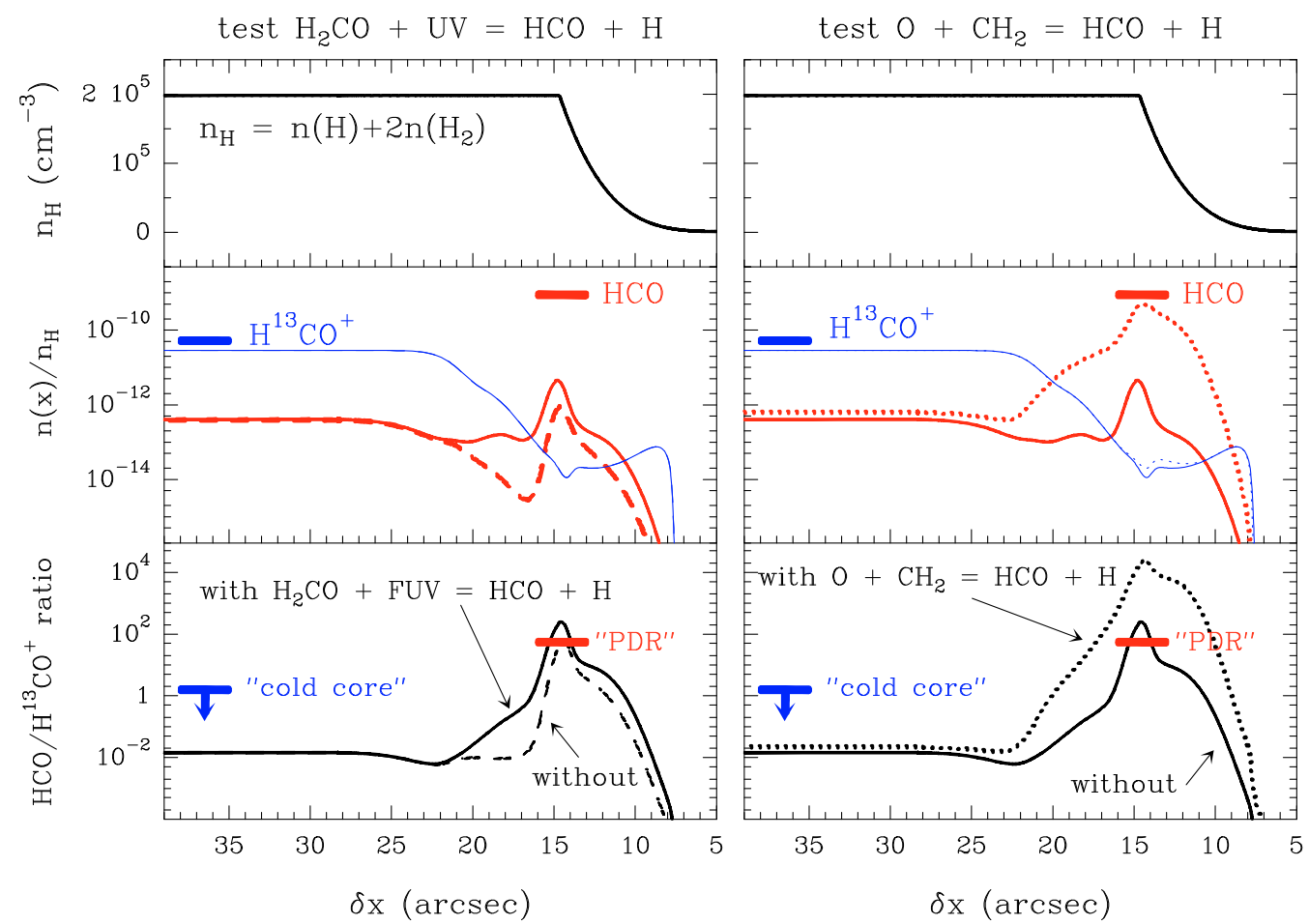

Fig. 4. Photochemical models of a unidimensional PDR. Upper panels show the density gradient $\left(n_{\mathrm{H}}=n(\mathrm{H})+2 n\left(\mathrm{H}_{2}\right)\right.$ in $\left.\mathrm{cm}^{-3}\right)$ used in the calculation. Middle panels show the predicted $\mathrm{HCO}$ and $\mathrm{H}^{13} \mathrm{CO}^{+}$abundances (relative to $n_{\mathrm{H}}$ ). The $\mathrm{H}^{13} \mathrm{CO}^{+}$abundance inferred from observations in the cold core ("the $\mathrm{DCO}^{+}$peak", see the offsets in Table 2) is shown with blue lines. The HCO abundance inferred from observations in the PDR ("the HCO peak", see the offsets in Table 2) is shown with red lines. Lower panels show the $\mathrm{HCO} / \mathrm{H}^{13} \mathrm{CO}^{+}$abundance ratio predicted by the models whereas the $\mathrm{HCO} / \mathrm{H}^{13} \mathrm{CO}^{+}$column density ratio inferred from observations is shown as blue arrows and red lines (for the cold core and PDR respectively). Each panel compares two different models: left-side models show a standard chemistry (dashed curves) versus the same network upgraded with the addition of the $\mathrm{H}_{2} \mathrm{CO}+$ photon $\rightarrow \mathrm{HCO}+\mathrm{H}$ photodissociation (solid curves). Right-side models show the previous upgraded standard model (solid curves) versus a chemistry that adds the $\mathrm{O}+\mathrm{CH}_{2} \rightarrow \mathrm{HCO}+\mathrm{H}$ reaction with a rate of $5.01 \times 10^{-11} \mathrm{~cm}^{3} \mathrm{~s}^{-1}($ dotted curves). The inclusion of the $\mathrm{O}+\mathrm{CH}_{2}$ reaction has almost no effect on $\mathrm{H}^{13} \mathrm{CO}^{+}$for the physical conditions prevailing in the Horsehead, but triggers an increases of the $\mathrm{HCO}$ abundance in the PDR by two orders of magnitude.

emission towards the "the $\mathrm{DCO}^{+}$peak" arises from the quiescent, cold and dense core, whereas $\mathrm{HCO}$, in the same line of sight, arises predominantly from the warmer and more turbulent outer cloud layers. We note that the presence of a foreground layer of more diffuse material $\left(A_{\mathrm{V}} \sim 2 \mathrm{mag}\right)$ was already introduced by Goicoechea et al. (2006) to fit the CS $J=2-1$ scattered line emission. The analysis of CO $J=4-3$ and $\mathrm{CI}^{3} P_{1}-{ }^{3} P_{0}$ maps led Philipp et al. (2006) to propose the presence of a diffuse envelope, with $A_{\mathrm{V}} \sim 2 \mathrm{mag}$, and which contributes to about half the mass of the dense filament traced by $\mathrm{C}^{18} \mathrm{O}$ and the dust continuum emission. The hypothesis of a surface layer of HCO is therefore consistent with previous modeling of molecular emission of the Horsehead.

We conclude 1) that $\mathrm{HCO}$ and $\mathrm{HCO}^{+}$have similar abundances in the PDR; and 2) that the $\mathrm{HCO}$ abundance drops by at least one order of magnitude between the dense and warm PDR region and the cold and shielded $\mathrm{DCO}^{+}$core.

\section{HCO chemistry}

\subsection{Gas-phase formation: PDR models}

In order to understand the $\mathrm{HCO}$ and $\mathrm{H}^{13} \mathrm{CO}^{+}$abundances and $\mathrm{HCO} / \mathrm{H}^{13} \mathrm{CO}^{+}$column density ratio inferred from observations, we have modeled the steady state gas phase chemistry at the Horsehead edge. The density distribution in the PDR is well represented by a density gradient $n_{\mathrm{H}}(\delta x) \propto \delta x^{4}$, where $\delta x$ is the distance from the edge towards the cloud interior and
$n_{\mathrm{H}}=n(\mathrm{H})+2 n\left(\mathrm{H}_{2}\right)$ (see the top panels of Fig. 4). The density reaches a constant $n_{\mathrm{H}}$ value of $2 \times 10^{5} \mathrm{~cm}^{-3}$ in an equivalent length of $10^{\prime \prime}$ Habart et al. (2005); Goicoechea et al. (2006). The cloud edge is illuminated by a FUV field 60 times the mean interstellar radiation field $\left(G_{0}=60\right.$ in Draine units). We used the Meudon PDR code ${ }^{2}$, a photochemical model of a unidimensional PDR (see Le Bourlot et al. 1993; Le Petit et al. 2006; Goicoechea \& Le Bourlot 2007, for a detailed description). Our standard chemical network is based on a modified version of the Ohio State University (osu) gas-phase network, updated for photochemical studies (see Goicoechea et al. 2006). It also includes ${ }^{13} \mathrm{C}$ fractionation reactions Graedel et al. (1982) and specific computation of the ${ }^{13} \mathrm{CO}$ photodissociation rate as a function of depth. The ionization rate due to cosmic rays in the models is $\zeta=5 \times 10^{-17} \mathrm{~s}^{-1}$. Following our previous work, we chose the following elemental gas phase abundances: $\mathrm{He} / \mathrm{H}=0.1, \mathrm{O} / \mathrm{H}=$ $3 \times 10^{-4}, \mathrm{C} / \mathrm{H}=1.4 \times 10^{-4}, \mathrm{~N} / \mathrm{H}=8 \times 10^{-5}, \mathrm{~S} / \mathrm{H}=3.5 \times 10^{-6}$, ${ }^{13} \mathrm{C} / \mathrm{H}=2.3 \times 10^{-6}, \mathrm{Si} / \mathrm{H}=1.7 \times 10^{-8}$ and $\mathrm{Fe} / \mathrm{H}=1.0 \times 10^{-9}$.

In Fig. 4, we investigate the main gas-phase formation routes for HCO in a series of models "testing" different pathways leading to the formation of $\mathrm{HCO}$. $\mathrm{HCO}$ and $\mathrm{H}^{13} \mathrm{CO}^{+}$predictions are shown in Fig. 4 (middle panels). In all models the $\mathrm{HCO}$ abundance peaks near the cloud surface at $A_{\mathrm{V}} \simeq 1.5\left(\delta x \simeq 14^{\prime \prime}\right)$ where the ionization fraction is high $\left(\mathrm{e}^{-} / \mathrm{H} \sim 5 \times 10^{-5}\right)$. Due to the low abundance of metals in the model (as represented by the low abundance of $\mathrm{Fe}$ ), the ionization fraction in the shielded

$\overline{2}$ Publicly available at http $: / /$ aristote.obspm.fr/MIS/ 

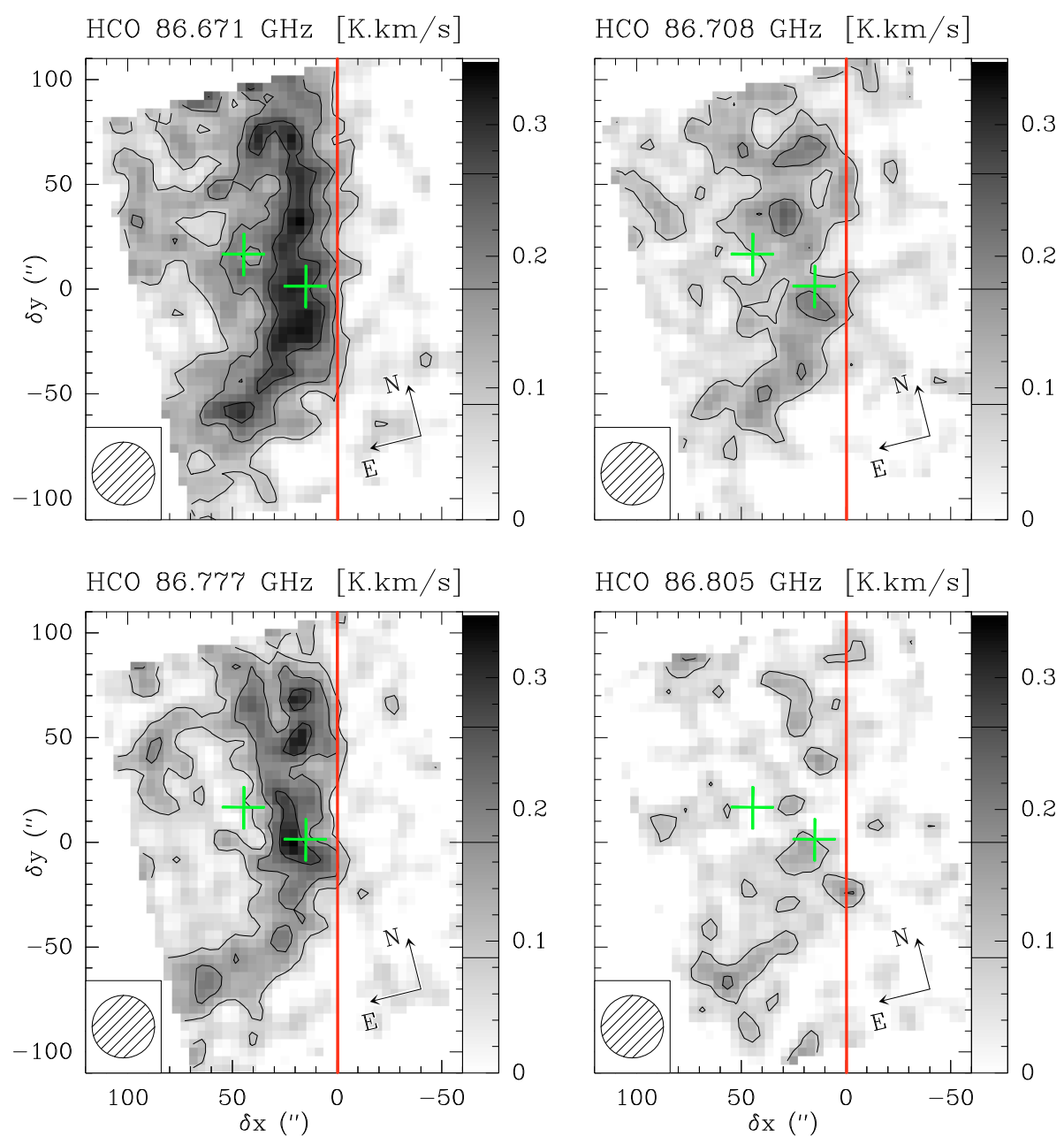

Fig. 5. Medium angular resolution maps of the integrated intensity of the 4 hyperfine components of the fundamental transition of HCO. These lines have been observed simultaneously at IRAM-30 m. Maps have been rotated by $14^{\circ}$ counter-clockwise around the projection center, located at $(\delta x, \delta y)=\left(20^{\prime \prime}, 0^{\prime \prime}\right)$, to bring the illuminated star direction in the horizontal direction and the horizontal zero has been set at the PDR edge. The emission of all lines is integrated between 9.6 and $11.4 \mathrm{~km} \mathrm{~s}^{-1}$. Displayed integrated intensities are expressed in the main beam temperature scale. Contour levels are displayed on the grey scale lookup tables. The red vertical line shows the PDR edge and the green crosses show the positions $\left(\mathrm{DCO}^{+}\right.$and $\mathrm{HCO}$ peaks) where deep integrations have been performed at IRAM-30 m (see Fig. 2).

regions is low $\left(\mathrm{e}^{-} / \mathrm{H} \lesssim 10^{-8}\right)$, and therefore the $\mathrm{H}^{13} \mathrm{CO}^{+}$predictions matches the observed values (Goicoechea et al. 2009). Besides, a low metalicity reduces the efficiency of charge exchange reactions of $\mathrm{HCO}^{+}$with metals, e.g.,

$\mathrm{Fe}+\mathrm{HCO}^{+} \rightarrow \mathrm{HCO}+\mathrm{Fe}^{+}$

which are the main gas-phase formation route of HCO in the FUV-shielded gas in our models. Hence, the HCO abundance remains low inside the core. Nevertheless, even though such models do reproduce the observed $\mathrm{HCO}$ distribution, which clearly peaks at the PDR position, the predicted absolute HCO abundances can vary by orders of magnitude depending of the dominant formation route.

In our standard model (left-side models: dashed curves), the formation of $\mathrm{HCO}$ in the PDR is dominated by the dissociative recombination of $\mathrm{H}_{2} \mathrm{CO}^{+}$, while its destruction is dominated by photodissociation. Even if the predicted $\mathrm{HCO} / \mathrm{H}^{13} \mathrm{CO}^{+}$abundance ratio satisfactorily reproduces the value inferred from observations, the predicted HCO abundance peak is $\sim 3$ orders of magnitude lower than observed. In order to increase the gasphase formation of the HCO in the PDR we have added a new channel in the photodissociation of formaldehyde, the production $\mathrm{HCO}$, in addition to the normal channel producing $\mathrm{CO}$ :

$\mathrm{H}_{2} \mathrm{CO}+$ photon $\rightarrow \mathrm{HCO}+\mathrm{H}$.

This channel is generally not included in standard chemical networks but very likely exists Troe (2007); Yin et al. (2007). We included this process with an unattenuated photodissociation rate of $\kappa_{\text {diss }}\left(\mathrm{H}_{2} \mathrm{CO}\right)=10^{-9} \mathrm{~s}^{-1}$ and a depth dependence given by $\exp \left(-1.74 A_{\mathrm{V}}\right)$. This is the same rate as the one given by van Dishoeck (1988) for the photodissociation of $\mathrm{H}_{2} \mathrm{CO}$ producing CO, which is explicitly calculated for the Draine (1978) radiation field. Model results are shown in Fig. 4 (left-side models: solid curves). The inclusion of Reaction 2, which becomes the dominant $\mathrm{HCO}$ formation route, increases the $\mathrm{HCO}$ abundance in the PDR by a factor of $\sim 5$. But the $\mathrm{HCO}$ production rate is still too low to reproduce the abundance determined from observations.

Another plausible possibility to increase the $\mathrm{HCO}$ abundance in the PDR by pure gas-phase processes is to include additional reactions of atomic oxygen with carbon radicals that reach high abundances only in the PDR. Among the investigated reactions, the most critical one,

$\mathrm{O}+\mathrm{CH}_{2} \rightarrow \mathrm{HCO}+\mathrm{H}$

is known to proceed with a relatively fast rate at high temperatures $\left(5.01 \times 10^{-11} \mathrm{~cm}^{3} \mathrm{~s}^{-1}\right.$ at $T_{\mathrm{k}}=1200-1800 \mathrm{~K}$; Tsuboi \& Hashimoto 1981). This is the rate recommended by NIST Mallard et al. (1994) and UMIST2006 Woodall et al. (2007) and that we adopt for our lower temperature domain $(\sim 10-200 \mathrm{~K})$. Model predictions are shown in Fig. 4 (right-side models: dotted curves). While the predicted HCO abundance in the shielded gas remains almost the same, the HCO abundance is dramatically increased in the PDR (by a factor of $\sim 125$ ) and the $\mathrm{O}+$ $\mathrm{CH}_{2}$ reaction becomes the $\mathrm{HCO}$ dominant production reaction. Therefore, such a pure gas-phase model adding reactions 2 and 3 not only reproduces the $\mathrm{H}^{13} \mathrm{CO}^{+}$abundance in the shielded core, 
but also reproduces the observed HCO absolute abundances in the PDR. In this picture, the enhanced HCO abundance that we observe in the Horsehead PDR edge would be fully determined by the gas-phase chemical path:

$$
\mathrm{C}^{+} \overrightarrow{\mathrm{H}_{2}} \quad \mathrm{CH}_{2}^{+} \quad \overrightarrow{\mathrm{H}_{2}} \quad \mathrm{CH}_{3}^{+} \quad \overrightarrow{\mathrm{e}} \quad \mathrm{CH}_{2} \quad \overrightarrow{\mathrm{o}} \mathrm{HCO} \text {. }
$$

The validity of the rate of Reaction 3 used in our PDR model remains, of course, to be confirmed theoretically or experimentally at the typical ISM temperatures (10 to $200 \mathrm{~K}$ ).

\subsection{Other routes for HCO formation: Grain photodesorption}

If Reaction 3 is not included in the chemical network, the predicted $\mathrm{HCO}$ abundance is $\sim 2$ orders of magnitude below the observed value towards the PDR. As a consequence, the presence of HCO in the gas-phase should be linked to grain mantle formation routes, and subsequent desorption processes (not taken into account in our modeling). In particular, Schilke et al. (2001) proposed that $\mathrm{HCO}$ could result from $\mathrm{H}_{2} \mathrm{CO}$ photodissociation, if large quantities of formaldehyde are formed on grain mantles and then released in the gas phase. Even with this assumption, their model could not reproduce the observed HCO abundance in highly illuminated PDRs such as the Orion Bar. The weaker FUV-radiation field in the Horsehead, but high density, prevent dust grains from acquiring high temperatures over large spatial scales. In fact, both gas and grains cool down below $\sim 30 \mathrm{~K}$ in $\simeq 10^{\prime \prime}-20^{\prime \prime}$ (or $A_{\mathrm{V}} \simeq 1-2$ ) as the FUV-radiation field is attenuated. Therefore, thermal desorption of dust ice-mantles (presumably formed before $\sigma$-Orionis ignited and started to illuminate the nebula) should play a negligible role. Hence a non-thermal desorption mechanism should be considered to produce the high abundance of $\mathrm{HCO}$ observed in the gas phase. This mechanism could either produce $\mathrm{HCO}$ directly or a precursor molecule such as formaldehyde.

Since high HCO abundances are only observed in the PDR, FUV induced ice-mantle photo-desorption (with rates that roughly scale with the FUV-radiation field strength) seems the best candidate (e.g., Willacy \& Williams 1993; Bergin et al. 1995). Laboratory experiments have shown that HCO radicals are produced in irradiated, methanol containing, ice mantles Bernstein et al. (1995); Moore et al. (2001); Bennett \& Kaiser (2007). The formyl radical could be formed through the hydrogenation of $\mathrm{CO}$ in the solid phase. It is an important intermediate radical in the synthesis of more complex organic molecules such as methyl formate or glycolaldehyde Bennett \& Kaiser (2007). However, the efficiency of the production of radicals in FUV irradiated ices remains uncertain, and very likely depends on the ice-mantle composition. The formation of species like formaldehyde and methanol in $\mathrm{CO}$-ice exposed to $\mathrm{H}$-atom bombardment has been reported by different groups Hiraoka et al. (1994); Watanabe et al. (2002); Linnartz et al. (2007), further confirming the importance of $\mathrm{HCO}$ as an intermediate product in the synthesis of organic molecules in ices. Indeed, hydrogenation reactions of $\mathrm{CO}$-ice, which form $\mathrm{HCO}, \mathrm{H}_{2} \mathrm{CO}, \mathrm{CH}_{3} \mathrm{O}$ and $\mathrm{CH}_{3} \mathrm{OH}$ in grain mantles (e.g., Tielens \& Whittet 1997; Charnley et al. 1997), are one important path which warrants further studies.

To compare with our observations, we further need to understand how the radicals are released in the gas phase, either directly during the photo-processing, or following FUV induced photo-desorption. Recent laboratory measurements have started to shed light on the efficiency of photo-desorption, which depends on the ice composition and molecule to be desorbed. For species such as CO, the rate of photo-desorbed molecules per FUV photon is much higher than previously thought (e.g., Öberg et al. 2007). Similar experiments are required to constrain the formation rate of the various species that can form in interstellar ices and to determine their photo-desorption rates.

We can use the measured gas phase abundance of $\mathrm{HCO}$ to constrain the efficiency of photo-desorption. We assume that the PDR is at steady state, and that the main $\mathrm{HCO}$ formation mechanism is non thermal photo-desorption from grain mantles (with a $F_{\text {HCO }}$ rate), while the main destruction mechanism is gas-phase photodissociation (with a $D_{\mathrm{HCO}}$ rate), therefore:

$$
\begin{aligned}
& D_{\mathrm{HCO}}=G_{0} \kappa_{\mathrm{diss}}(\mathrm{HCO}) \chi(\mathrm{HCO}) n\left(\mathrm{H}_{2}\right) \quad\left[\mathrm{cm}^{-3} \mathrm{~s}^{-1}\right] \\
& F_{\mathrm{HCO}}=G_{0} \kappa_{\mathrm{pd}}(\mathrm{HCO}) \chi\left(\mathrm{HCO}_{\text {ice }}\right) \frac{n\left(\mathrm{H}_{2} \mathrm{O}_{\text {ice }}\right)}{n\left(\mathrm{H}_{2}\right)} n\left(\mathrm{H}_{2}\right)\left[\mathrm{cm}^{-3} \mathrm{~s}^{-1}\right]
\end{aligned}
$$

where $\chi(\mathrm{HCO})$ is the gas phase abundance of $\mathrm{HCO}$ relative to $\mathrm{H}_{2}, \chi\left(\mathrm{HCO}_{\text {ice }}\right)$ is the solid phase abundance relative to water ice, and $n\left(\mathrm{H}_{2} \mathrm{O}_{\text {ice }}\right) / n\left(\mathrm{H}_{2}\right)$ is the fraction of water in the solid phase relative to the total gas density. $\kappa_{\text {diss }}(\mathrm{HCO})$ and $\kappa_{\mathrm{pd}}(\mathrm{HCO})$ are the $\mathrm{HCO}$ photodissociation and photo-desorption rates respectively.

By equaling the formation and destruction rates, we get:

$\kappa_{\mathrm{pd}}(\mathrm{HCO})=\kappa_{\text {diss }}(\mathrm{HCO}) \frac{\chi(\mathrm{HCO})}{\chi\left(\mathrm{HCO}_{\text {ice }}\right)} \frac{n\left(\mathrm{H}_{2}\right)}{n\left(\mathrm{H}_{2} \mathrm{O}_{\text {ice }}\right)} \quad\left[\mathrm{s}^{-1}\right]$

or

$\frac{\kappa_{\mathrm{pd}}(\mathrm{HCO})}{\mathrm{s}^{-1}} \approx 10^{-12} \frac{\kappa_{\mathrm{diss}}(\mathrm{HCO})}{10^{-9}} \frac{\chi(\mathrm{HCO}) / 10^{-9}}{\chi\left(\mathrm{HCO}_{\text {ice }}\right) / 10^{-2}} \frac{10^{-4} n\left(\mathrm{H}_{2}\right)}{n\left(\mathrm{H}_{2} \mathrm{O}_{\text {ice }}\right)}$

where we have used typical figures for the $\mathrm{HCO}$ abundance in the gas phase $\left(\sim 10^{-9}\right.$, see above $)$ and solid phase $\left(\sim 10^{-2}\right.$ see e.g. Bennet \& Kaiser 2007) and for the amount of oxygen present as water ice in grain mantles.

Assuming standard ISM grains with a radius of $0.1 \mu \mathrm{m}$ the required photodesorption efficiency (or yield) $Y_{\mathrm{pd}}(\mathrm{HCO})$ :

$Y_{\mathrm{pd}}(\mathrm{HCO}) \simeq \frac{\kappa_{\mathrm{pd}}(\mathrm{HCO})}{G_{0} \exp \left(-2 A_{\mathrm{V}}\right) \pi a^{2}} \quad\left[\right.$ molecules photon $\left.^{-1}\right]$

(see e.g., d'Hendecourt et al. 1985; Bergin et al. 1995) converts to $Y_{\mathrm{pd}}(\mathrm{HCO}) \approx 10^{-4}$ molecules per photon (for the FUV radiation field in the Horsehead and $A_{\mathrm{V}} \simeq 1.5$, where HCO peaks). Therefore, the production of HCO in the gas phase from photodesorption of formyl radicals could be a valid alternative to gas phase production, if the photo-desorption efficiency is high and HCO abundant in the ice mantles. This mechanism also requires further laboratory and theoretical studies.

Because the formyl radical is closely related to formaldehyde and methanol and the three species are likely to coexist in the ice mantles, a combined analysis of the $\mathrm{H}_{2} \mathrm{CO}, \mathrm{CH}_{3} \mathrm{OH}$ and HCO line emissions towards the Horsehead nebula (PDR and cores) is needed to provide more information on the relative efficiencies of gas-phase and solid-phase routes in the formation of complex organic molecules in environments dominated by FUVradiation. This will be the subject of a future paper.

\section{Summary and conclusions}

We have presented interferometric and single-dish data showing the spatial distribution of the formyl radical rotational lines in the Horsehead PDR and associated dense core. The HCO emission delineates the illuminated edge of the nebula and coincides with the $\mathrm{PAH}$ and hydrocarbon emission. $\mathrm{HCO}$ and $\mathrm{HCO}^{+}$ reach similar abundances $\left(\simeq 1-2 \times 10^{-9}\right)$ in these PDR regions 
where the chemistry is dominated by the presence of FUV photons. For the physical conditions prevailing in the Horsehead edge, pure gas-phase chemistry is able to reproduce the observed $\mathrm{HCO}$ abundances (high in the PDR, low in the shielded core) if the $\mathrm{O}+\mathrm{CH}_{2} \rightarrow \mathrm{HCO}+\mathrm{H}$ reaction is included in the models. This reaction connects the high abundance of $\mathrm{HCO}$, through its formation from carbon radicals, with the availability of $\mathrm{C}^{+}$in the PDR.

The different linewidths of $\mathrm{HCO}$ and $\mathrm{H}^{13} \mathrm{CO}^{+}$in the line of sight towards the "DCO ${ }^{+}$peak" suggest that the $\mathrm{H}^{13} \mathrm{CO}^{+}$line emission arises from the quiescent, cold and dense gas completely shielded from the FUV radiation, whereas HCO predominantly arises from the outer surface of the cloud (its illuminated skin). As a result we propose the $\mathrm{HCO} / \mathrm{H}^{13} \mathrm{CO}^{+}$abundance ratio, and the HCO abundance itself (if $\gtrsim 10^{-10}$ ), as sensitive diagnostics of the presence of FUV radiation fields. In particular, regions where the $\mathrm{HCO} / \mathrm{H}^{13} \mathrm{CO}^{+}$abundance ratio (or intensity ratio if lines are optically thin) is greater than $\simeq 1$ should reflect ongoing FUV-photochemistry.

Given the rich HCO spectrum and the possibility of mapping its bright millimeter line emission with interferometers, we propose $\mathrm{HCO}-\mathrm{H}_{2}$ as a very interesting molecular system for calculations of the ab initio inelastic collision rates.

Acknowledgements. We thank the IRAM PdBI and $30 \mathrm{~m}$ staff for their support during the observations. We thank A. Faure and J. Tennyson for sending us the $\mathrm{H}^{13} \mathrm{CO}^{+}-\mathrm{e}^{-}$collisional rates prior to publication, B. Godard for useful discussions on the chemistry of carbon ions in the diffuse ISM, and A. Bergeat and A. Canosa for interesting discussions on radical-atom chemical reactions. J.R.G. is supported by a Ramón y Cajal research contract from the Spanish MICINN and co-financed by the European Social Fund. This research has benefitted from the financial support of the CNRS/INSU research programme, PCMI. We acknowledge the use of the JPL (Pickett et al. 1998) and Cologne (Müller et al. 2001, 2005) spectroscopic data bases, as well as the UMIST chemical reaction data base Woodall et al. (2007)

\section{References}

Abergel, A., Bernard, J. P., Boulanger, F., et al. 2002, A\&A, 389, 239 Bennett, C. J., \& Kaiser, R. I. 2007, ApJ, 661, 899

Bergin, E. A., Langer, W. D., \& Goldsmith, P. F. 1995, ApJ, 441, 222

Bernstein, M. P., Sandford, S. A., Allamandola, L. J., Chang, S., \& Scharberg, M. A. 1995, ApJ, 454, 327

Charnley, S. B., Tielens, A. G. G. M., \& Rodgers, S. D. 1997, ApJ, 482, L203

d'Hendecourt, L. B., Allamandola, L. J., \& Greenberg, J. M. 1985, A\&A, 152 130

Draine, B. T. 1978, ApJS, 36, 595

de Jong, T., Boland, W., \& Dalgarno, A. 1980, A\&A, 91, 68

Flower, D. R. 1999, MNRAS, 305, 651
García-Burillo, S., Martín-Pintado, J., Fuente, A., Usero, A., \& Neri, R. 2002, ApJ, 575, L55

Goicoechea, J. R., \& Le Bourlot, J. 2007, A\&A, 467, 1

Goicoechea, J. R., Pety, J., Gerin, M., et al. 2006, A\&A, 456, 565

Goicoechea, J. R., Pety, J., Gerin, M., et al. 2009, A\&A, in press

González-García, M., Le Bourlot, J., Le Petit, F., \& Roueff, E. 2008, A\&A, 485, 127

Graedel, T. E., Langer, W. D., \& Frerking, M. A. 1982, ApJS, 48, 321

Habart, E., Abergel, A., Walmsley, C. M., Teyssier, D., \& Pety, J. 2005, A\&A, 437, 177

Hiraoka, K., Ohashi, N., Kihara, N., et al. 1994, Chem. Phys. Lett., 229, 408

Landsberg, B. M., Merer, A. J., \& Oka, T. 1977, J. Mol. Spec., 67, 459

Le Bourlot, J., Pineau Des Forets, G., Roueff, E., \& Flower, D. R. 1993, A\&A, 267, 233

Le Petit, F., Nehmé, C, Le Bourlot, J., \& Roueff, E. 2006, ApJS, 64, 506

Linnartz, H., Acharyya, K., Awad, Z., et al. 2007, Molecules in Space and Laboratory, ed. J. L. Lemaire, \& F. Combes

Mallard, et al. 1994, NIST Chemical Kinetics Database, NIST, Gaithersburg, MD

Moore, M. H., Hudson, R. L., \& Gerakines, P. A. 2001, Spec. Acta part A, 57, 843

Müller, H. S. P., Thorwirth, S., Roth, D. A., \& Winnewisser, W. 2001, A\&A, 370, L49

Müller, H. S. P., Schlöder, F., Stutzki, J., \& Winnewisser, W. 2005, J. Mol. Struct., 742,215

Öberg, K. I., Fuchs, G. W., Awad, Z., et al. 2007, ApJ, 662, L23

Penzias, A. A., \& Burrus, C. A. 1973, ARA\&A, 11, 51

Pety, J., Teyssier, D., Fossé, D., et al. 2005, A\&A, 435, 885

Pety, J. SF2A-2005: Semaine de 1'Astrophysique Française, meeting held in Strasbourg, France, ed. F. Casoli, T. Contini, J. M. Hameury, \& L. Pagani (EDP Sciences, Conf. Ser.), 721

Pety, J., Goicoechea, J. R., Hily-Blant, P., Gerin, M., \& Teyssier, D. 2007a, A\&A, 464, L41

Pety, J., Goicoechea, J. R., Gerin, M., et al. 2007b, Proceedings of the Molecules in Space and Laboratory conference, ed. J. L. Lemaire, \& F. Combes

Philipp, S. D., Lis, D. C., Güsten, R., et al. 2006, A\&A, 454, 213

Pickett, H. M., Poynter, R. L., Cohen, E. A., et al. 1998, J. Quant. Spectrosc. Rad. Transfer, 60, 883

Röllig, M., et al. 2007, A\&A, 467, 187

Schilke, P., Pineau des Forets, G., Walmsley, C. M., \& Martín-Pintado, J. 2001, A\&A, 372, 291

Snyder, L. E., Hollis, J. M., \& Ulich, B. L. 1976, ApJ, 208, L91

Snyder, L. E., Schenewerk, M. S., \& Hollis, J. M. 1985, ApJ, 298, 360

Teyssier, D., Fossé, D., Gerin, M., et al. 2004, A\&A, 417, 135

Tielens, A. G. G. M., \& Whittet, D. C. B. 1997, in Molecules in astrophysics: probe and processes, ed. E. F. van Dishoeck (Dordrecht: Kluwer), 45

Troe, J. 2007, J. Phys. Chem., 111, 3868

Tsuboi, T., \& Hashimoto, K. 1981, J. Combust. Flame, 42

van Dishoeck, E. F. 1988, Rate Coefficients in Astrochemistry, ed. T. J. Millar,

\& D. A. Williams (Dordrecht, Boston: Kluwer Academic Publishers), 49

Watanabe, N., \& Kouchi, A. 2002, ApJ, 571, L173

Willacy, K., \& Williams, D. A. 1993, MNRAS, 260, 635

Woodall, J., Agúndez, M., Markwick-Kemper, A. J., \& Millar, T. J. 2007, A\&A, 466, 1197

Yin, H. M., Rowling, S. T., Büll, A., \& Kable, S. H. 2007, J. Chem. Phys., 064302 